\title{
The Physico-Chemical Properties of Lipids during the Development and Regression of Atherosclerosis*
}

\author{
Prof. Donald M. SMALL**
}

I want to share with you the people who have helped me with this work. I have had collaborators, Robert Lees from MIT, DeWitt Goodman from Columbia, M. Gene Bond from Bowman Bray and E.H. Ahrens from the Rockefeller, and a number of very good graduate students and research fellows and in particular Saul Katz who carried out much of the early and important work that I am going to talk about today. Most of the work I am going to talk about has been summarized in my Duff Lecture to the American Heart Association (Small D.M. "Progression and Regression of Atherosclerotic Lesions: Insights from Lipid Physical Biochemistry" Arteriosclerosis, 8: 109-129, 1988).

What I am going to cover today are the following questions:

- What is the lipid content and composition of normal and atherosclerotic intima?

- What is the physical behavior of intimal lipids?

- What are the states of lipids in normal and atherosclerotic intima?

- Can we separate the different lipid phases from plaques?

- How rapidly does the cholesterol exchange between the lipid phases of plaques and plasma?

- Where are the lipid phases in the anatomy of the lesion?

- How do plaques form and grow?

- Do plaques regress? If so, how?

The pathogenesis of atherosclerosis involves the development of a space occupying lesion in the intima of major arteries. The process may progress to stenosed lumen of the artery and cause sym-

* This paper was presented at the 21 st annual meeting of the Japan Atherosclerosis Society in June 1989 in Tokyo

** Department of Biophysics, Boston University School of Medicine, Boston ptoms or may ultimately thrombose to cause a myocardial infarction. We will concentrate on the development well before thrombosis.

\section{I) Lipid Content and Composition of Arterial Intima}

The lipid composition of the intima of arteries is shown in Fig. 1. You can see that there is a small amount of lipid in normal intima which increases with age. Fatty streaks on the other hand contain about $30 \%$ lipid of the dry weight and atheroma contain about $60 \%$ lipid. Thus lipids are an important part of the plaque contributing largely to its volume and to its space occupying characteristics.

The three major lipid classes which accumulate in atherosclerotic lesions are cholesterol, cholesterol esters such as cholesterol linoleate, and phospholipids, mainly lecithins and sphingomyelins.

\section{Method of Plotting Composition by Using Triangular Coordinates}

Now, the compositions of any three components can be plotted on triangular coordinates, in which each component is at the apex of the triangle (Fig. 2). Mixtures of two components lie along the side and any point within the surface of the triangle is a mixture of all three components. So that $45 \% \mathrm{C}, 20 \% \mathrm{PL}$, and $35 \% \mathrm{CE}$ would have the point $\mathbf{P}$. A mixture containing equal amounts of all three would fall in the center of the triangle.

Consider now how the addition and subtraction of a component changes the composition position. If we subtract cholesterol ester from this composition, then the composition will move away from the cholesterol ester apex. If we add free cholesterol the point will move towards the 


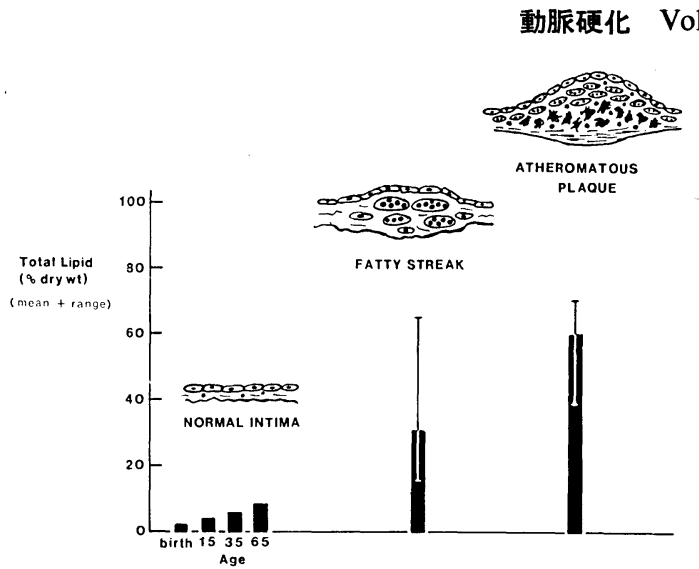

Fig. 1 The lipid content of intima and atherosclerotic lesions given as the mean percentage of the dry weight of intimal tissue plus or minus the range of determinations. In normal intima the lipid content increases from about $2 \%$ at birth to about $7 \%$ to $8 \%$ by age 65 ; this lipid is largely extracellular. Fatty streaks have more lipid, and the atheroma of plaques are rich in lipid.

cholesterol apex; and if we were to subtract phospholipid, it would move away from the phospholipid apex; if we added phospholipid, it would move towards the phospholipid apex (Fig. 3).

What happens if we mix two components together (Fig. 4)? If we mix equal parts of $\mathrm{A}$ and $\mathrm{B}$ together (Fig. 4), then the mixture will lie half way on a line connecting the composition $\mathrm{A}$ and the composition $\mathrm{B}$, and so forth.

\section{Normal Newborn Intima Con- trasted to Plaque Composition}

Now, let's go back to the old literature and plot the composition of plaques and of normal intima from a very young children (Fig. 5). The composition of the intima is very rich in phospholipid and contains a small amount of free cholesterol and virtually no cholesterol ester. The composition of the plaques, however, contains only about $20-30 \%$ phospholipid and is very rich in free cholesterol and in cholesterol ester. Thus during the development of the atherosclerotic plaque, not only is the absolute lipid content greatly increased but the lipid composition changes from phospholipid rich to cholesterol/cholesterol ester rich. To be able to interpret the compositional changes found between normal intima and plaques we need to understand the physical be-

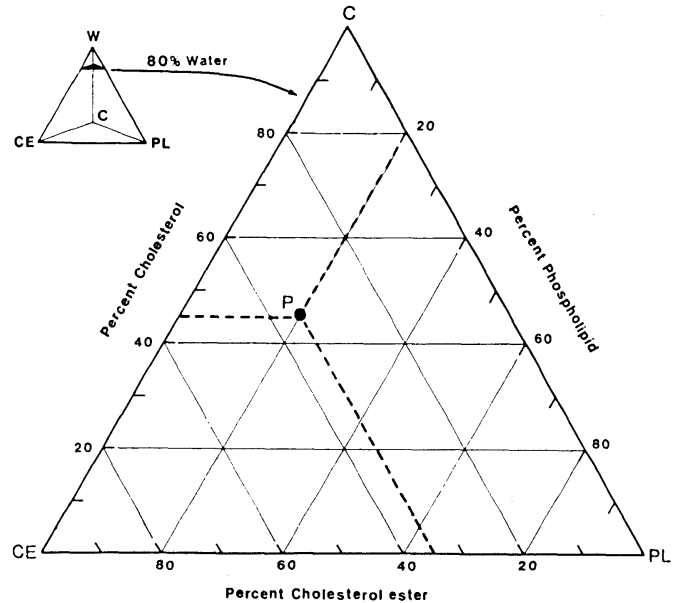

Fig. 2 The method of representing cholesterol (C), phospholipid (PL), and cholesterol ester (CE) compositions on triangular coordinates. The true system is C, PL, CE, and water and would be represented by a regular tetrahedron (upper left). However, by fixing water as an excess component (for instance at $80 \%$ ) the lipid system can be expressed as a three-component system (C, PL, CE) at constant water. In fact, the water content of intima probably varies between $50 \%$ and $80 \%$. The percentage total weights of $\mathrm{CE}, \mathrm{PL}$, and $\mathrm{C}$ constituted by each of these components are shown on the scales along the side of the triangle. Since the sum of $\mathrm{CE}, \mathrm{PL}$, and $\mathrm{C}$ equals $100 \%$, the composition of any mixture containing these components can be represented as a single point within the triangular coordinates. Thus, a mixture containing $45 \% \mathrm{C}, 20 \% \mathrm{PL}$, and $35 \% \mathrm{CE}$ would be represented by point $\mathrm{P}$ at the intersection of the dashed lines extended from the $35 \%$ level on the CE scale at the base of the triangle, the $20 \%$ level on the PL scale at the right, and the $45 \%$ level on the $\mathrm{C}$ scale at the left. All triangles will be represented as $w \mathrm{t} \%$ in this paper although mol $\%$ may also be used.

havior of the intimal lipids.

\section{Physical Behavior and Interactions of Major Lipids of the Intima}

The individual molecules are shown in Fig. 6. Above are the chemical formulas, and in the middle are molecular cartoons, and at the bottom are the physical states of the individual molecules in water. All these lipids are insoluble in water. Free cholesterol forms crystals, cholesterol mono- 


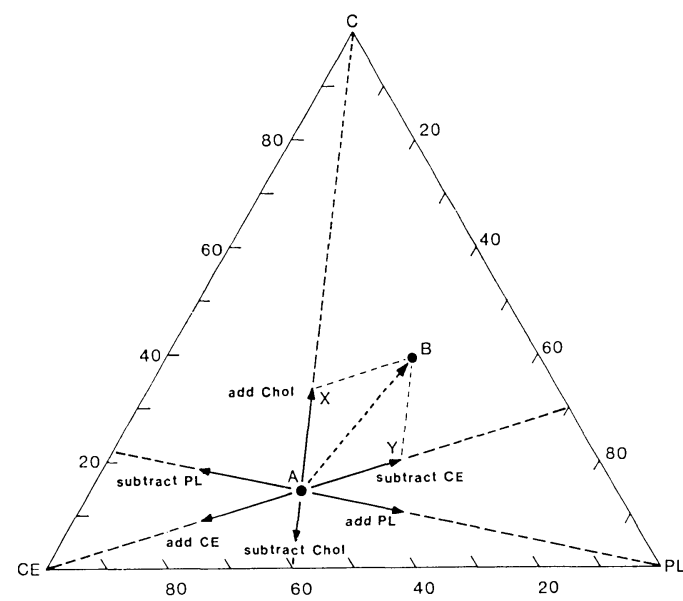

Fig. 3 The effect of adding or subtracting single components to or from a given composition. If $\mathrm{CE}$ is subtracted from the composition marked by point $\mathrm{A}(14.5 \% \mathrm{C}, 34 \% \mathrm{PL}, 51.5 \% \mathrm{CE})$, the composition will move directly away from the $\mathrm{CE}$ apex toward the intersection of the C-PL axis on the right. The ratio of $\mathrm{C}$ to $\mathrm{PL}$ will remain the same $(30 \% \mathrm{C}, 70 \% \mathrm{PL})$ along this line because only CE is subtracted. Adding CE to such a mixture will move the composition toward the $C E$ apex leaving the $C$ to $P L$ ratio unchanged. Similar explanations apply to the addition and subtraction of free $\mathrm{C}$ and the addition and subtraction of PL (see arrows). If the composition moves in a direction that is not from along one of the lines connecting $A$ to an apex, then a combination of two or more changes occurs. For instance, if point A moved to point $B$, then the compositional change would be represented by two vectors, one moving along the line $\mathrm{AX}$ indicating an addition of free $\mathrm{C}$ and one moving along line AY indicating a subtraction of $\mathrm{CE}$.

hydrate crystals, that melts at $85^{\circ} \mathrm{C}$. Phospholipid such as lecithins and sphingomyelins form bilayers and swell in water. And cholesterol esters form oils or smectic liquid crystals, depending upon the acyl chain which is esterified to the cholesterol. To understand interactions between these molecules over a period of years we made over 1,000 separate mixtures, examined their physical state by a number of techniques, plotted the states on triangular coordinates to arrive at a phase diagram describing the interaction of these three lipid classes.

The distillate of this work is shown in Fig. 7. There are four zones: two one-phase zones, a two-

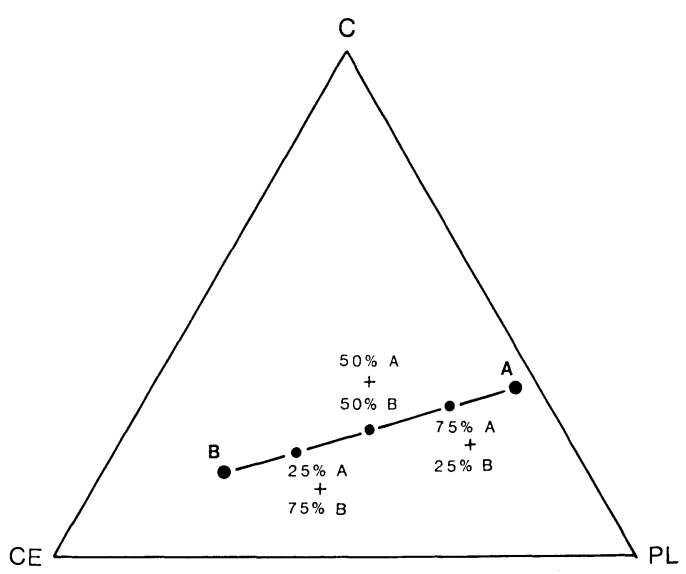

Fig. 4 The effects of adding one composition or mixture to another. If composition B is added to composition $\mathrm{A}$, then the mixtures will fall along the line connecting the two. The amount of $\mathbf{B}$ added to $\mathrm{A}$ is reflected by the position along the line $\mathrm{AB}$. The amount of $\mathrm{B}$ added to $A$ is inversely proportional to the distance along line $\mathrm{AB}$; for example, when $25 \% \mathrm{~B}$ is added to $75 \% \mathrm{~A}$, the representative point is $1 / 4$ the distance from $A$ to $B$.

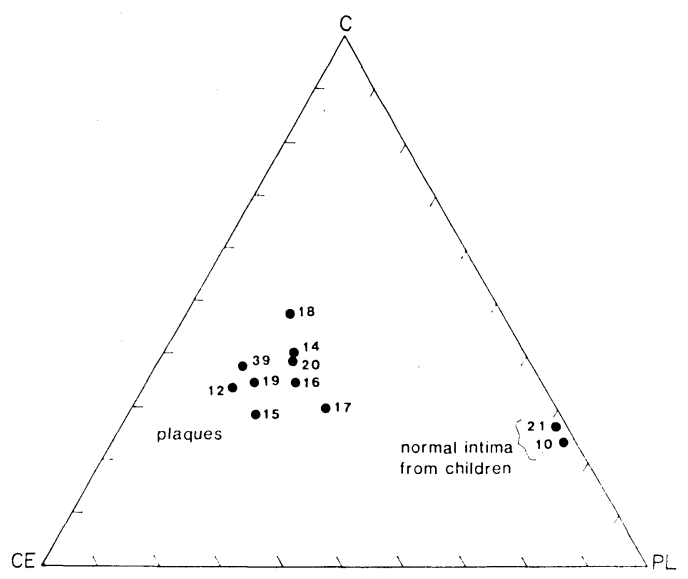

Fig. 5 The composition of normal intima contrasted to atherosclerotic plaques. The numbers by the points refer to the references from which the data were derived.

phase zone, and a three-phase zone. A phase is a homogeneous, physically distinct, mechanically separable state of matter, such as a liquid, a liquid crystal, or a solid. Zone II consists of a single phase of cholesterol esters containing a small amount of free cholesterol. This can be present in 


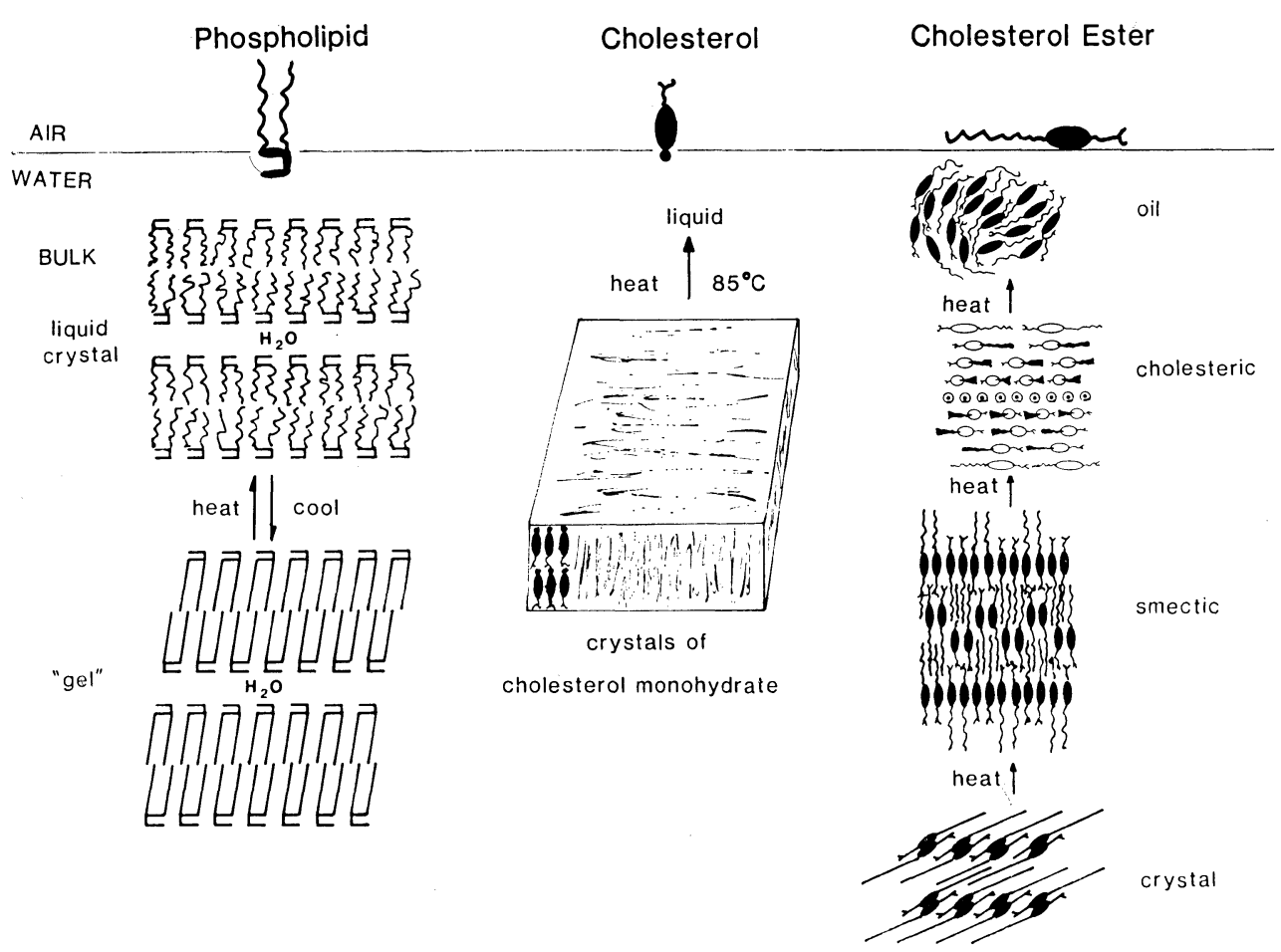

Fig. 6 The physical states of phospholipids (PL), cholesterol (C), and cholesterol esters (CE) at the air-water interface and in bulk systems. PL form stable monolayers at air-water and oil-water interfaces. They are insoluble in oil and in water and congregate at interfaces. The term PL in this context applies specifically to mixtures of phospholipids occurring in the intima and in atherosclerotic lesions; that is, to mixtures rich in phosphatidylcholine and sphingomyelin and poor in other PL. C also forms a stable monolayer but has limited solubility in oil phases such as $\mathrm{CE}$ or triacylglycerol ( 3 to $5 \mathrm{wt} \%$ at room temperature). $\mathrm{C}$ has a very low solubility in water $\left(\sim 3 \times 10^{-8} \mathrm{M}\right)$. CE do not form stable monolayers and separate from the surface as oily droplets or crystals. In bulk aqueous systems, PL that are above their chain melting transition (above the solidification of the acyl moieties) form a lamellar liquid crystal in which bilayers of PL molecules are separated by layers of water. On cooling, such lipids may form a gel in which the chains become solid. $\mathrm{C}$ crystallizes in an aqueous system to form cholesterol monohydrate crystals, which melt at $85^{\circ} \mathrm{C}$ and reform as anhydrous cholesterol crystals. They have a characteristic morphology and may be easily recognized in nonfixed preparations. CE may form four or more separate states that are separated from the aqueous phase. These states depend on the temperature and the acyl chain esterified through the sterol. At higher temperatures, an oily liquid is formed; on cooling a cholesteric liquid crystalline phase may be formed; on further cooling, a layered smectic liquid crystalline phase may be formed; and finally, a solid crystalline phase may be formed.

a liquid state shown as a randomly oriented molecules or as an ordered liquid crystalline state with a periodicity of about 35 angstroms. Zone I is another single phase region, the phospholipid bilayered or membrane-like phase which consists of bilayers of phospholipid into which cholesterol and a very small amount of cholesterol ester can be incorporated. This membrane bilayer phase becomes saturated with cholesterol at 33 weight $\%$ or about 1 molecule of cholesterol per molecule 


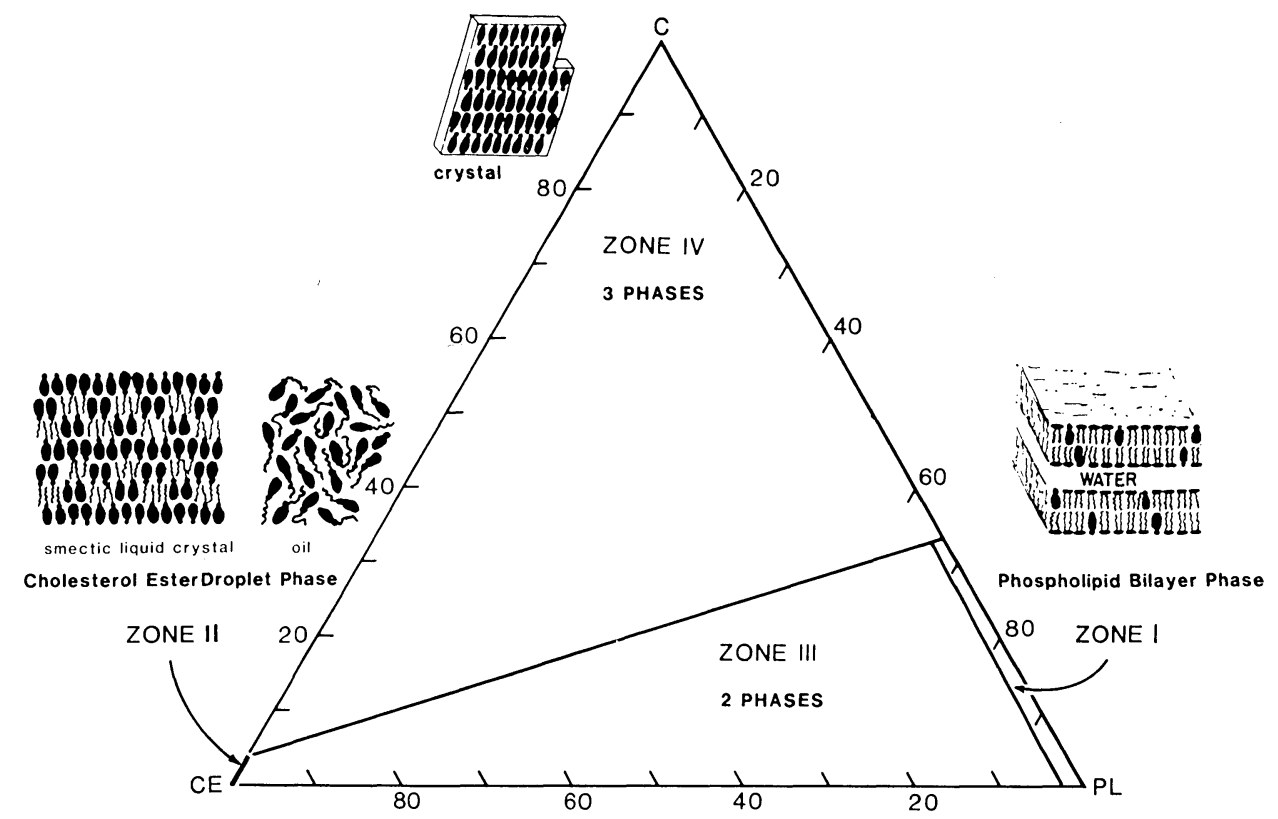

Fig. 7 Phase diagram of a three-component system showing interactions of cholesterol (C), phospholid (PL), and cholesterol ester (CE) in excess water at $37^{\circ} \mathrm{C}$ and 1 atmosphere pressure. Zone I contains a single phospholipid lamellar liquidcrystal phase, into which $33 \% \mathrm{C}$ and $2 \% \mathrm{CE}$ can be incorporated. Zone II also contains a single phase, composed of liquid or liquid-crystalline CE. Zone III contains both the CE and PL phases. In Zone IV a third phase, cholesterolmonohydrate crystals, is also present. Schematic molecular representation of the phases is shown near each apex of the triangle. The irregular lines denote the PL molecule; the solid symbols, the C molecule; and the solid tailed symbols, CE.

of phospholipid. The zone between these two phases, zone III consists of mixtures of the two phases. Thus, both membrane-like phases and oily-like phases of cholesterol ester rich molecules will be found in this zone, and they can be separated by centrifugation, since the oily phase is less dense than the membrane phase. Finally, in this large region, region IV, three phases coexist at equilibrium; a cholesterol crystal phase, an oily cholesterol-ester phase saturated with free cholesterol, and a membrane phase saturated with cholesterol.

Having looked at the molecular structure of the phases, it is important to note that we can identify these stages, these phases, by the hotstage polarizing microscope in picogram quantities.

As shown in Fig. 8, the crystals have a distinct morphology with an edge angle of 79 degrees. They are flat plates and they melt at $85^{\circ} \mathrm{C}$. The choles- terol ester phase can be present as an oil or an ordered liquid crystal. The oil is non-birefringent under the polarizing microscope, and appears black, whereas a liquid crystal has birefringence and appears as Maltese crosses. The ester content of the droplet determines the melting point. The more saturated, the higher the melting point. Thus, at body temperatures, cholesterol esters rich in linoleate will be in the oil phase and appear black, while those rich in oleate or saturates like palmitate will have higher melting points and be in the liquid crystalline state and appear as Maltese crosses. Finally the lamellar phase of phospholipid also forms droplets, which appear as Maltese crosses and myelin figures but their melting point is very high compared to cholesterol esters, and thus we can distinguish between cholesterol ester and membrane phases by changing the temperature. 
動脈硬化 Vol. 18 No. 61990

Identification of Tissue Liplds by Hot Stage Polarizing Microscopo

\begin{tabular}{|c|c|c|c|c|}
\hline Lipid & $\begin{array}{c}\text { State at } \\
\text { Body Temperature }\end{array}$ & Morphology & $\begin{array}{l}\text { Thermal } \\
\text { Behavior }\end{array}$ & $\begin{array}{l}\text { ral Llpid } \\
\text { aining }\end{array}$ \\
\hline Cholesterol Monohydrate & Crystal & & melt $>85^{\circ} \mathrm{C}$ & No \\
\hline \multicolumn{5}{|l|}{ Cholesterol Esters } \\
\hline rich in Oleate and Palmitate & Liquid Crystal & & $\begin{array}{l}\text { melt to liquid } \\
\text { at } 40-55^{\circ} \mathrm{C} \text {,reversible }\end{array}$ & Yes \\
\hline rich in Linoleate & Oil & & $\begin{array}{l}\text { form } \because 8 \\
\text { on cooling to } 37-20^{\circ} \mathrm{C}\end{array}$ & Yes \\
\hline $\begin{array}{l}\text { Triglycerides } \\
\text { more saturated }\end{array}$ & Crystal & & $\begin{array}{l}\text { melt on heating } \\
\text { crystallize after } \\
\text { undercooling }>20^{\circ} \mathrm{C}\end{array}$ & No \\
\hline more unsaturated & Oil & & $\begin{array}{l}\text { crystallize after } \\
\text { undercooling }<20^{\circ} \mathrm{C}\end{array}$ & Yes \\
\hline $\begin{array}{l}\text { Phospholipids and } \\
\text { Complex Membrane Lipids }\end{array}$ & $\begin{array}{l}\text { Liquid Crystal } \\
\text { multilamellar } \\
\text { llposomes } \\
\text { vesicles } \\
\text { membranes }\end{array}$ & & melt $>85^{\circ} \mathrm{C}$ & No \\
\hline
\end{tabular}

Fig. 8 Identification of tissue lipids by hot-stage polarizing microscope. The major lipids of biological importance can be identified in fresh, unfixed, unstained, hydrated tissue samples by hot-stage polarizing microscope. Cholesterol monohydrate crystals such as occur in gallstones, atherosclerosis, and other necrotic deposits have typical plate-like morphology with acute edge angles of $79^{\circ} \mathrm{C}$. They melt to a liquid crystalline form at about $85^{\circ} \mathrm{C}$. Mixtures of cholesterol esters rich in saturated and monounsaturated acyl moieties tend to be liquid crystalline at body temperature and to melt at between $40^{\circ}$ and $55^{\circ} \mathrm{C}$. The liquid crystals are present as Maltese crosses under crossed polarized light. When mixtures of esters are rich in polyunsaturates, such as cholesteryl linoleate, they are liquid at body temperature and form Maltese crosses only at lower temperatures. Triglycerides do not form liquid crystals but instead form needle-like crystalline structures. While most triglyceride deposits are liquid in vivo, they can crystallize when cooled below their melting points. The undercooling and lack of liquid crystalline phases is characteristic of triglycerides. The more saturated the triglyceride, the higher the melting point. Mixtures of phospholipids or complex membrane lipids generally form a variety of bilayered structures that are strictly liquid crystalline but may be present as multilamellar liposomes, unilamellar vesicles, or membrane fragments. Multilamellar liposomes appear as spherical or tubular processes (myelin figures). The spherical particles exhibit a Maltese cross under under crossed polarized light and are indistinguishable from cholesterol ester droplets except that their melting points are much higher $\left(>85^{\circ} \mathrm{C}\right)$.

\section{States of Lipids in Normal and Atherosclerotic Intima}

Having determined the interactions between lipids, we wish to know what are the states of the lipids in the normal and atherosclerotic intima.

In a series of important experiments Saul Katz determined the liquid composition and physical state of 86 normally appearing intimas, taken from subjects from birth to old age. The mean compositions are shown by decades as the points on the graph of Fig. 9. The normal intima of new-borns or in the first decade is found in the zone occupied by the phospholipid bilayer phase and thus can be thought of as a phase of normal cell membranes containing phospholipids and a moderate amount of cholesterol. As the intima ages, it accumulates cholesterol ester. As it accumulates cholesterol ester it moves into zone III, the cholesterol ester rich phase separates and is also present. Physically the cholesterol ester is present in small 
The Physico-Chemical Properties of Lipids during the Development and Regression of Atherosclerosis

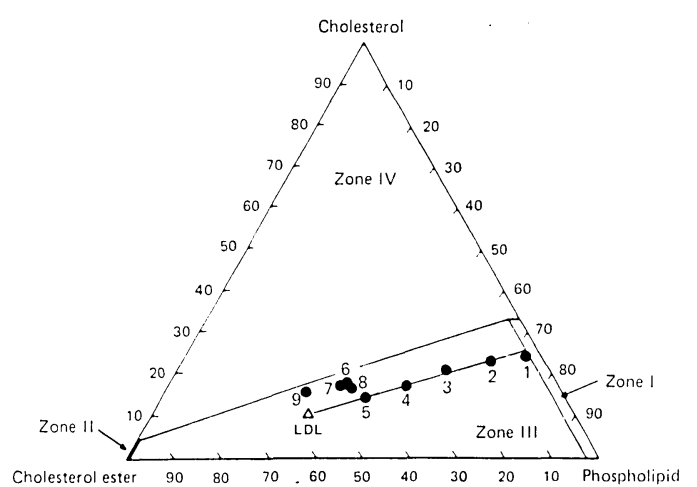

Fig. 9 The composition of normal intimal lipids of newborns and persons in subsequent decades of age as plotted on the cholesterol-phospholipid-cholesterol ester phase diagram (see Fig. 7). Points 1 to 9 represent intimal lipids gathered from subjects of increasing age. Intimal lipids and compositions up to the fourth decade fall on a straight line joining the newborn composition to low density lipoprotein (LDL). This indicates that LDL-like lipids are added to the newborn intimal composition as it ages. Refer to Fig. 4.

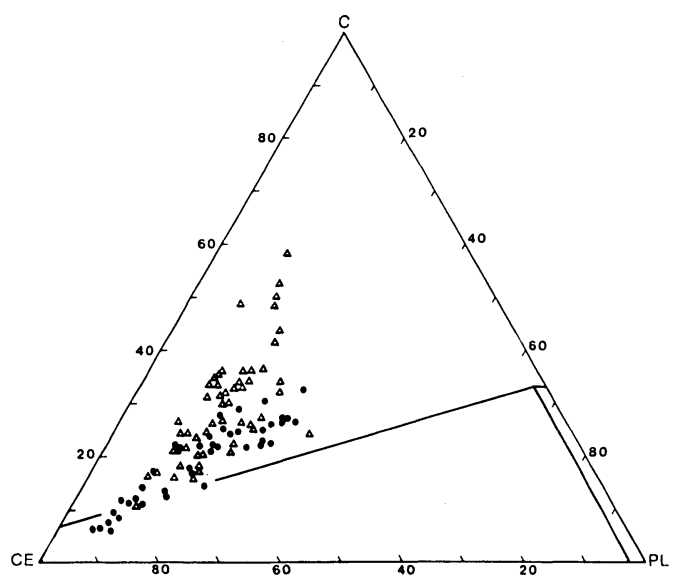

Fig. 10 The composition of individual intimal human atherosclerotic lesions. - fatty streaks, $\Delta=$ plaques.

extracellular particles of 200-1,000 angstroms in diameter. To the fifth decade the composition proceeds inexorably towards the composition of low density lipoprotein, as if low-density-lipoprotein-like lipids were being added or deposited in the extracellular space of the artery. In later decades there is an increase in the free cholesterol,

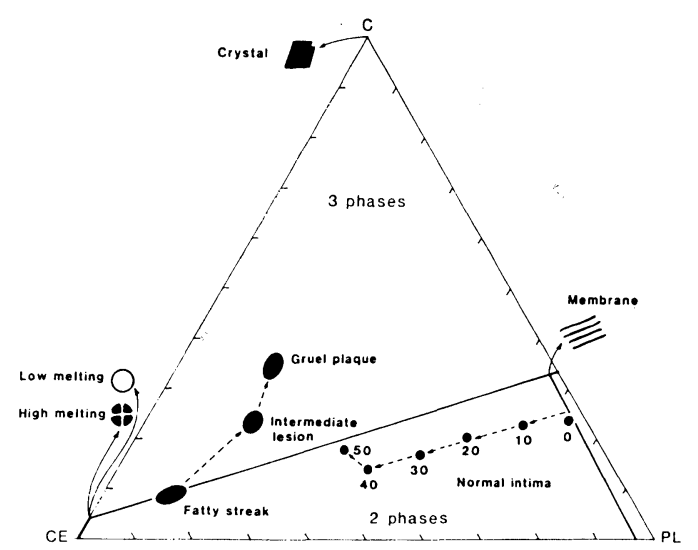

Fig. 11 The composition of normal intima contrasted to the mean composition of the fatty streak lesion, the intermediate lesion, and the gruel plaque. The size of the black oval dots giving the composition of the lesions indicates the mean \pm 1 standard error of the mean.

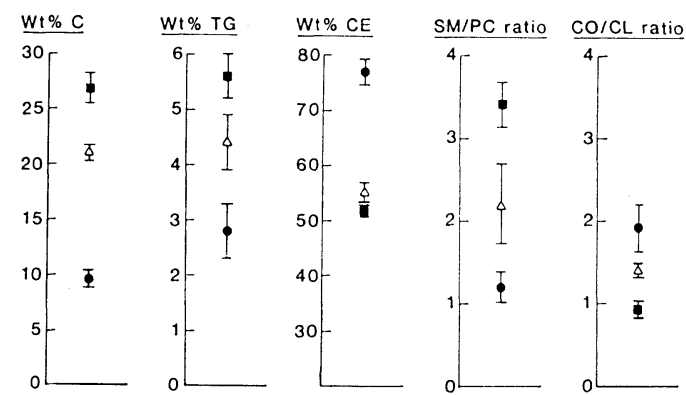

Fig. 12 Comparison of the chemical composition of fatty streaks $(\bullet)$, intermediate lesions $(\Delta)$, and plaques $(\boldsymbol{\square})$.

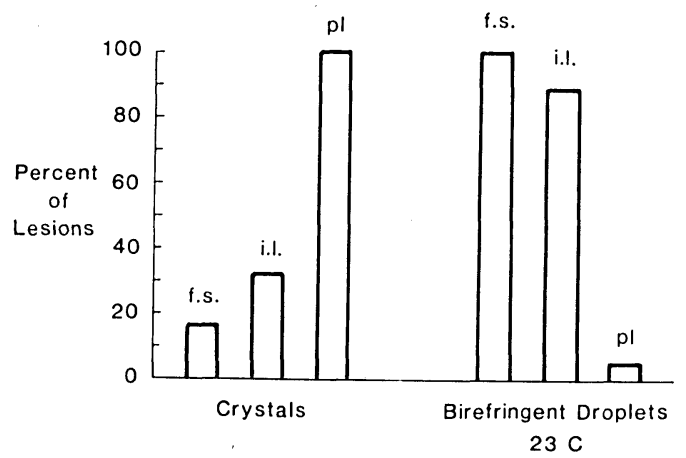

Fig. 13 The frequency of crystals and birefringent droplets at $23^{\circ} \mathrm{C}$ in fatty streaks (f.s.), intermediate lesions (i.l.), and plaques (pl). 


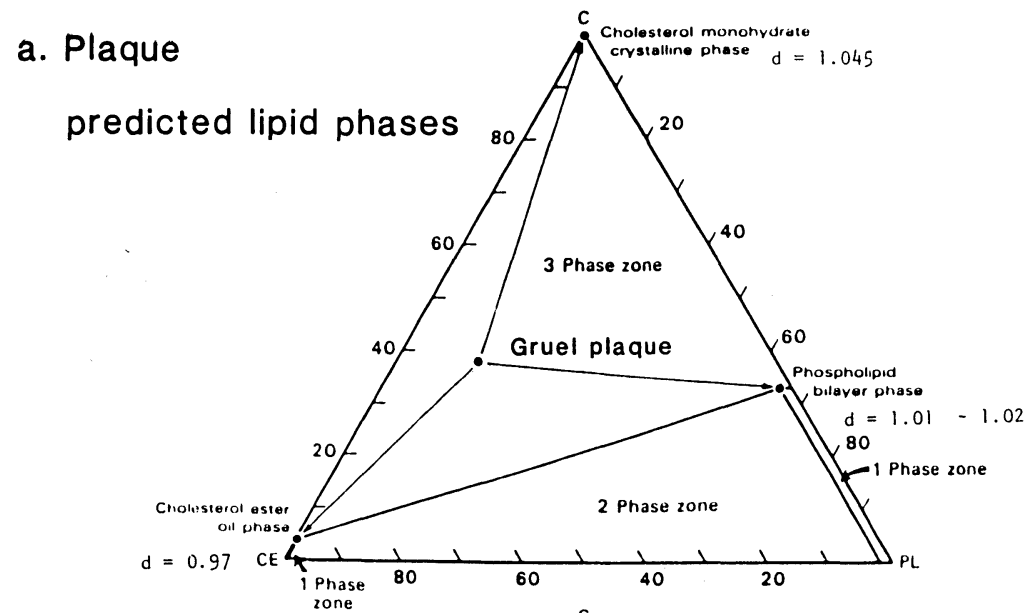

\section{b. Isolated Plaque Lipids}

phases isolated

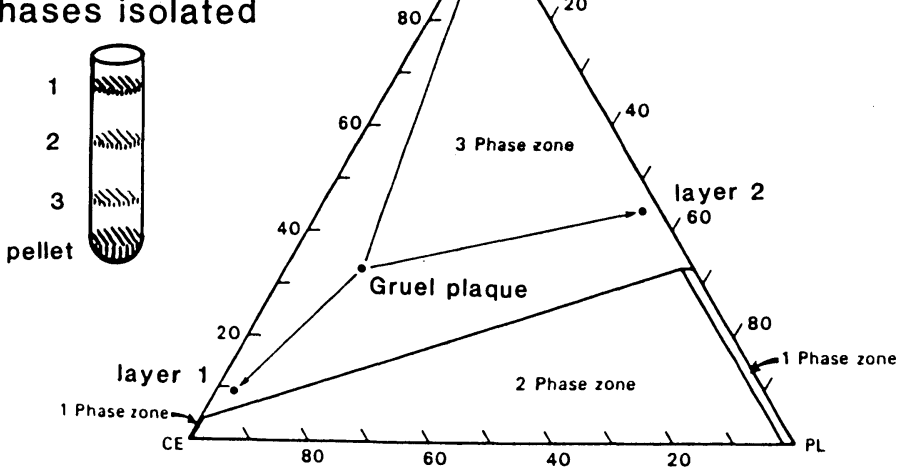

\section{c. Plaque}

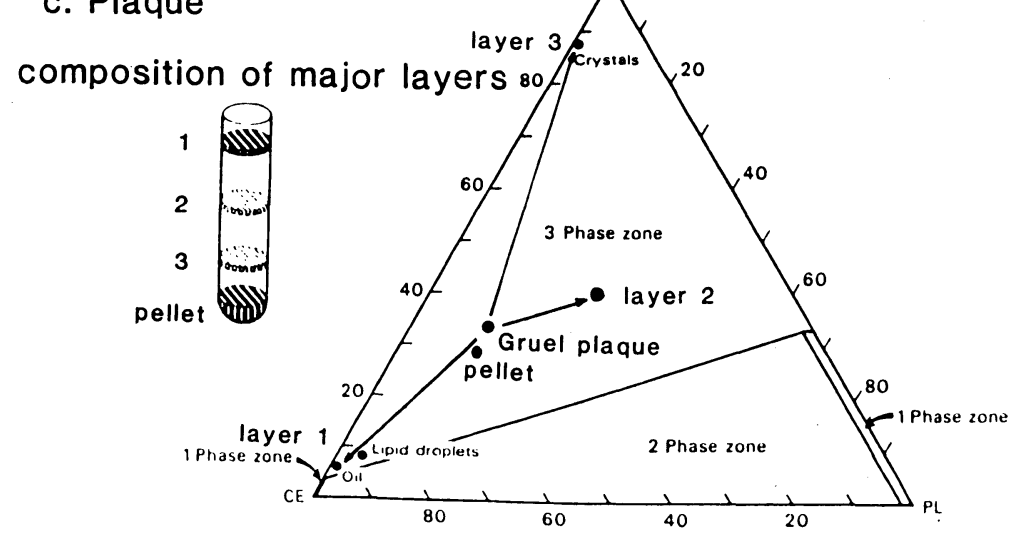

Fig. 14 Isolated lipid fractions of plaque. A. Average lipid composition of gruel plaques plotted on the phase diagram of cholesterol (C), cholesterol ester (CE), and phospholipid (PL). Arrows indicate the composition of the three coexisting lipid phases of the plaques as predicted by the phase diagram. B. Composition of layers of density gradient separation of lipids extracted from gruel plaques. C. Lipid composition of layers of plaque homogenates separated by density gradient centrifugation as in $\mathbf{B}$. Schematic representations of the layers of the gradients are shown on the left. 
and the content of the intimal lipid moves towards the line of saturation with free cholesterol. The importance of this change is not yet understood.

Now, let's turn to the composition of the two grossly identifiable types of atherosclerotic lesions, that is, fatty streaks and plaques.

Figure 10 shows the composition of over 100 separate individual lesions dissected by Dr. Katz and me from fresh human arteries. You can see that they are very different from the composition of the normal intima which is shown in Fig. 9. There is a continuum between fatty streaks shown as circles and plaques shown as triangles. There are indeed fatty streaks which do not overlap the composition of plaques, and plaques which do not overlap the composition of fatty streaks. But there is a lot of overlap between their compositions.

We have divided the fatty streak lesions into: 1) those lesions which would have less cholesterol than would saturate them. That is, these lesions which lie on or below the line describing the saturation with free cholesterol. These lesions we call ordinary fatty streaks. And 2) those lesions above the line (the round circles above the line in Fig. 10) which are indistinguishable from ordinary fatty streaks by gross examination, but are actually intermediate or transitional lesions between ordinary fatty streaks and plaques.

Figure 11 gives the mean composition of the fatty streaks which are less than saturated, intermediate lesions and plaques. You will note that the fatty streak lesion is extremely rich in cholesterol esters and not very rich in free cholesterol. The intermediate lesion has more free cholesterol and less ester and the plaque has still more free cholesterol. If one dissects the intima from a fresh, living fatty streak and puts it on a slide en face and looks at it under crossed polarized light without fixing or staining it, one will see an en face view of the living fatty streak shows stellate foam cells filled with birefringent cholesterol ester droplets 1-2 microns in diameter.

If we break open those cells as Lang and Insull and Dr. Hata and his colleagues did many years ago, one will see that each droplet shows a Maltese cross which melt at the appropriate temperature for cholesterol esters.

Now, let's move to the intermediate lesion.

The intermediate lesion looks grossly like the fatty streak, and microscopically has very little necrosis, but has chemical characteristics and physical characteristics different from plaques and from ordinary fatty streaks (Fig. 12, Fig. 13).

The Fig. 12 shows that intermediate lesions are indeed intermediate between fatty streaks and plaques. They have an intermediate amount of free cholesterol, triglyceride, cholesterol ester. The sphingomyelin/phosphatidylcholine ratio is also intermediate and the cholesteryl oleate to cholesteryl linoleate ratio is intermediate.

However, as for the physical properties, there are very few cholesterol crystals in ordinary fatty streaks and only about $30 \%$ of the intermediate lesions have crystals, whereas 100 percent of plaques contain cholesterol monohydrate crystals (Fig. 13). The birefringent droplets look very much like a fatty streak at $25^{\circ} \mathrm{C}$ but not very much like a plaque, because most of the droplets in the plaque are melted at $25^{\circ} \mathrm{C}$. So, these lesions appear to be a "pre-atheroma" in Stary's terminology or a "transitional" lesion in McGill's terminology. We call them intermediate because they are intermediate or transitional between the fatty streak and the atheromatous plaque. On a physical-chemical basis, they are cholesterol-supersaturated fatty streaks whose potential metastability renders them poised to nucleate and grow cholesterol crystals and thus to become necrotic and develop into an atheroma.

The atheromatous plaque has a composition which has more free cholesterol (Fig. 11) and all of the plaques contain cholesterol monohydrate crystals. If one squashes the core of an atheroma on a slide and looks at it under the polarizing microscope, one sees that not only birefringent droplets but also there are a profusion of crystals. These crystals have the characteristic edge angles of $79^{\circ}$ and birefringence of cholesterol monohydrate and they give X-ray diffraction patterns identical to authentic cholesterol monohydrate. Some of these crystals in human atheroma can be as long as a millimeter. Thus a hallmark of plaques are cholesterol monohydrate crystals.

Well, if the plaque contains three phases can we separate the different lipid phases from plaques?

In theory the plaque composition shown in Fig. 14a should consist of three phases. An oil saturated with cholesterol ester, a membrane phase saturated with free cholesterol, and cholesterol monohydrate crystals.

We worked out a technique using both discontinuous density gradient centrifugation and 


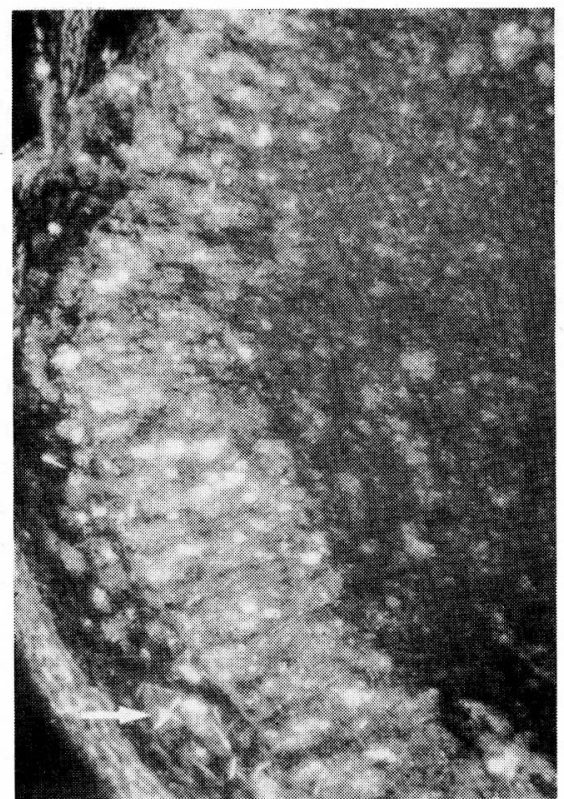

A

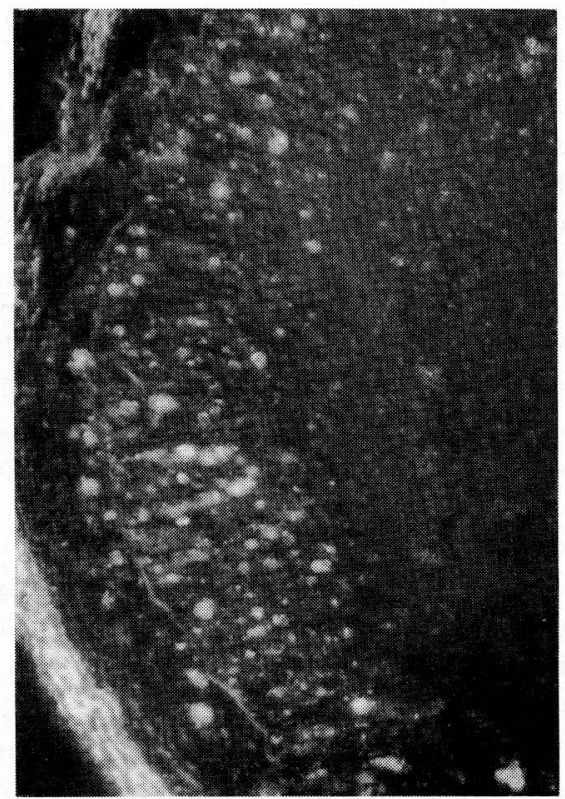

C

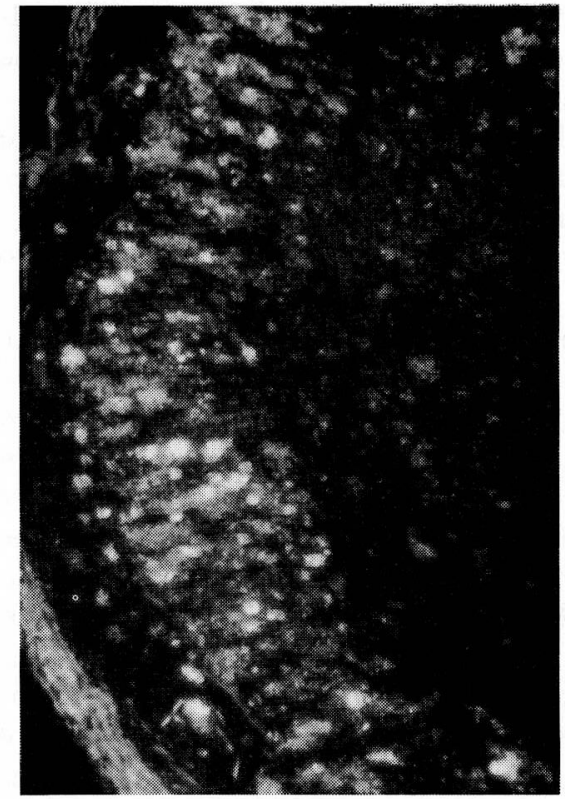

B

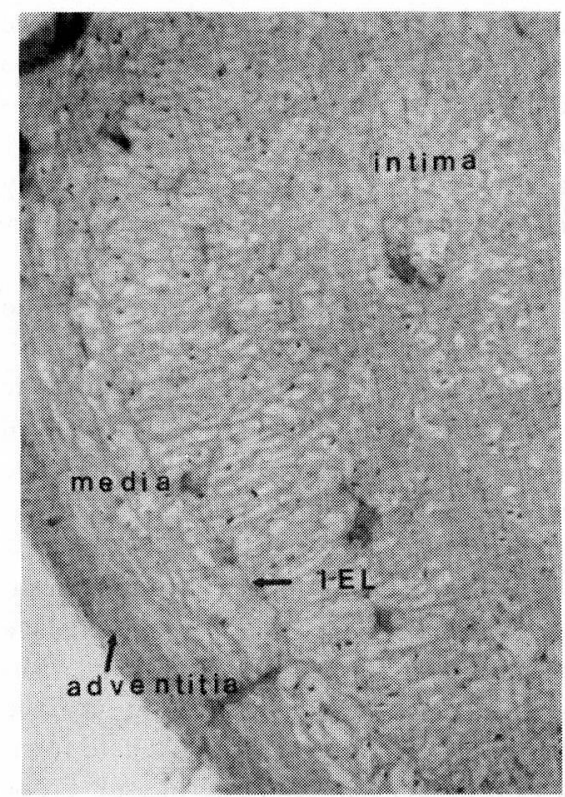

D

Fig. 15 Melting sequence of foam cells in an atherosclerotic lesion of a cholesterol-fed rabbit. Cells were viewed under polarized light. A. At $25^{\circ} \mathrm{C}$ birefringent foam cells are aligned along the internal elastic lamina (IEL). Foam cells are also present in the media and intima nearer the lumen. In addition, crystals can be seen in the media (arrow). Birefringent material in the adventitia is connective tissue (collagen and elastin). B. At $41^{\circ} \mathrm{C}$ the cellular birefringence is about half muted, consistent with liquid crystal melting, but connective tissue birefringence is unchanged. Crystals in the media are also melting. C. By $45^{\circ} \mathrm{C}$ most of the cells and crystals have melted, but there is a differential melting of the cells; that is, some cells appear to melt before others. Since the crystals have all melted by $45^{\circ} \mathrm{C}$, they cannot be cholesterol monohydrate (see Fig. 8). On cooling they form liquid crystals and, therefore, are cholesterol esters. The connective tissue birefringence remains unchanged. D. Same section stained with hematoxylin and eosin after healting. $\times 128$. 


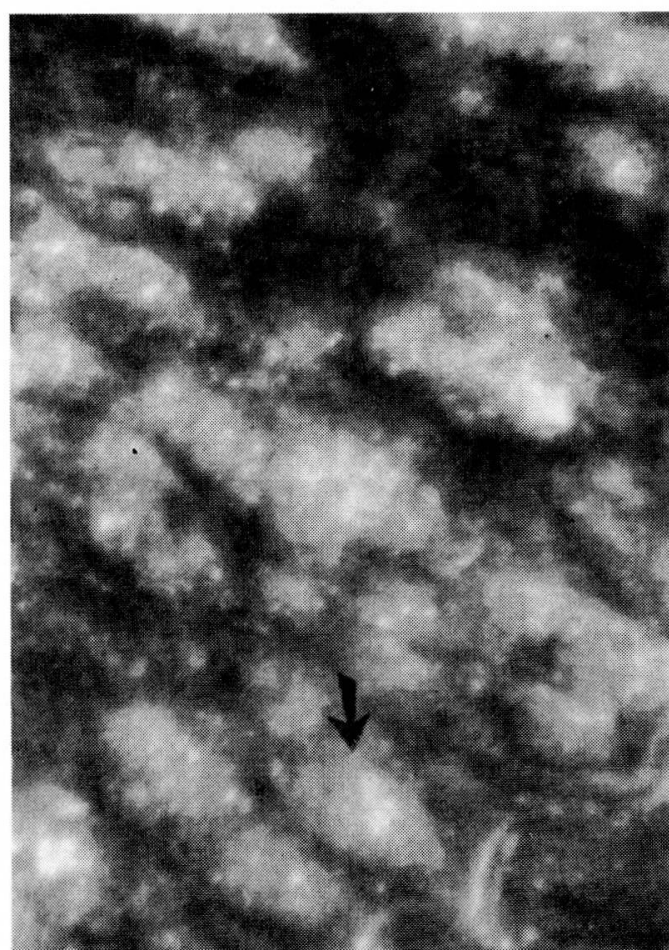

A

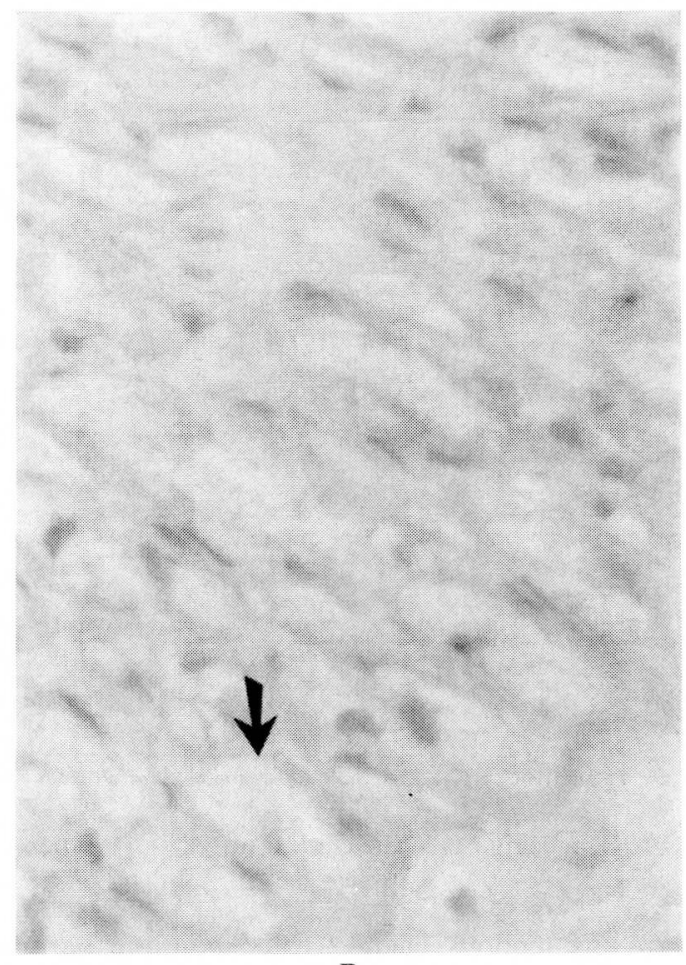

B

Fig. 16 Section of atherosclerotic iliac artery viewed under ploarized light before staining (A) and under bright-field illumination after staining (B). Corresponding cells can be identified in each section (arrow) enabling correlations between histology and the lipid characteristics of foam cells. Internal elastic lamina is at upper right-hand corner. Fig. 21 B, hematoxylin and eosin, $\times 400$.

continuous gradient centrifugation, to isolate cholesterol ester droplet phase, the phospholipid bilayers, the cholesterol monohydrate crystals, and a pellet which would contain protein, nucleic acids and other things at the bottom of the tube. The compositions of those isolated fractions are shown in Fig. 14.

The oily cholesterol ester phase could be obtained in a quite pure fashion as shown in Fig. 14. The cholesterol monohydrate phase could also be obtained with a little absorbed cholesterol ester on it but quite pure, but the membrane phase was not pure and was always contaminated with crystals and with some cholesterol esters that we could not clearly separate.

The pellet has the composition of the pre-existing plaque and contained about $20 \%$ of the total lipid, the other $80 \%$ being found in these three phases.

Having isolated the different phases in a rela- tively pure form we had a chance to ask the question: How rapidly does cholesterol exchange between these phases and plasma? Quite early, Chobanian and Hollander and Wm. Connor and his group had isolated cholesterol from plaques of patients who have been given radio-active cholesterol to obtain cholesterol pool sizes. They noted that the specific activity of the plaque cholesterol was usually low compared to that with plasma and suggested that the cholesterol turnover rate was slow in plaques. With the collaboration of DeWitt Goodman and his colleagues Dr. Katz and I were able to obtain plaques from two patients who had been given two separate doses of radio-active cholesterol at different times. This allowed us to estimate the exchange rate of cholesterol between plasma and the isolated plaque phases.

To summarize briefly, the exchange of unesterified cholesterol out of the oily phase was 


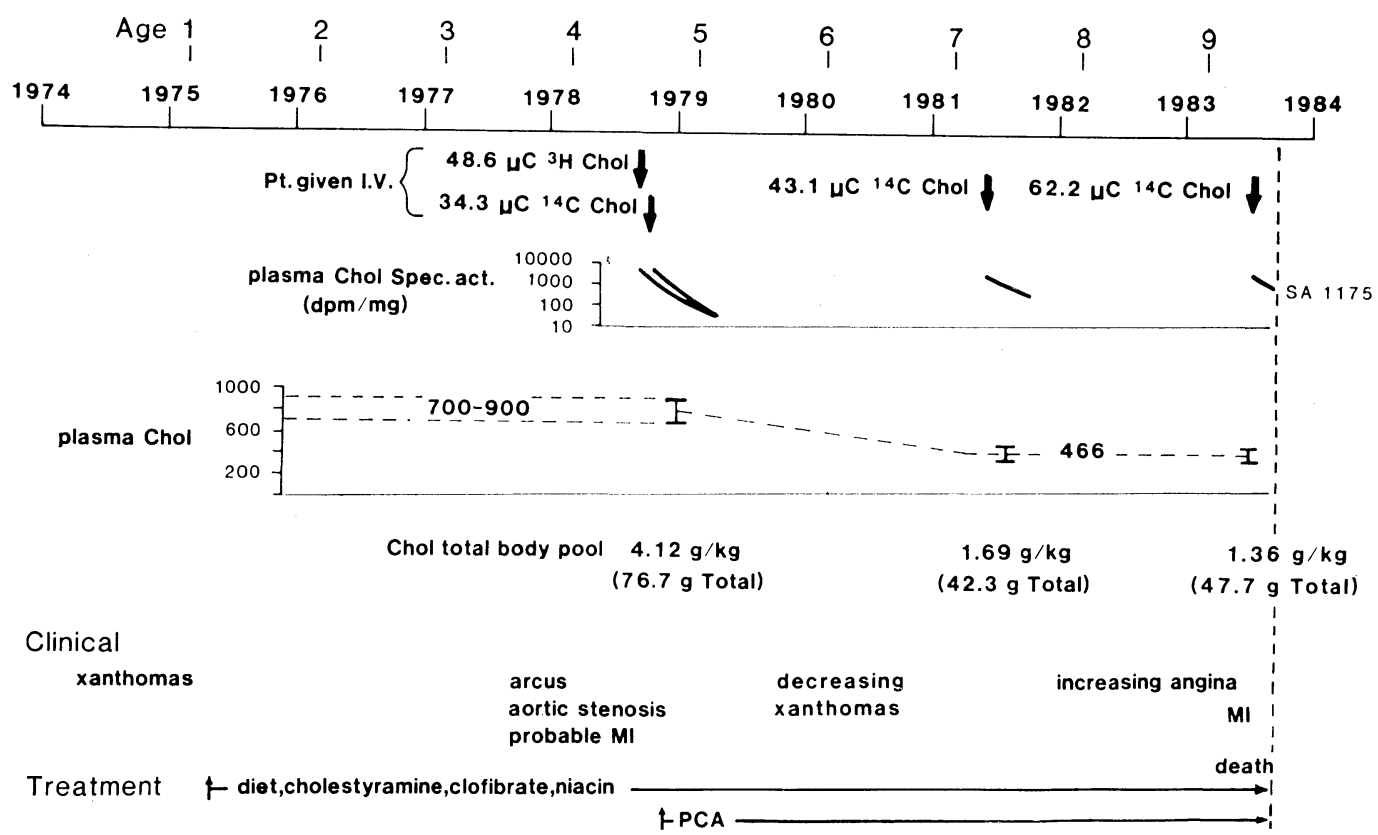

Fig. 17 The clinical history of a patient with familial homozygous hypercholesterolemia.

slow like tendons being 2 or 3 months; the exchange out of membrane phase was somewhat slower but still on the order of months. We were never able to obtain an equilibrium in the cholesterol crystals and therefore the exchange rate must have been extremely slow, certainly greater than six months which was the end time of our experiment. Thus free cholesterol turns over slow, quite slow or extremely slowly. The cholesterol ester in the oily phase turns over with about the same time constant as the free cholesterol in the oily phase. And thus the cholesterol ester as a whole turn over more rapidly than the free cholesterol.

\section{Localization of the Lipid Phases in the Anatomy of the Plaque}

Because of the physical state of the lipids within the atherosclerotic lesions and because the turnover rate of the cholesterol molecules within them was of the order of months or even years, the question arose: Where are the lipid phases in the anatomy of the lesion? Where are these droplets and crystals in relation to the anatomy of the lesion itself? That is, how are these studies related to the actual histology of the lesion?

Since standard histological methods extract lipids, and staining frozen sections with fat stains, such as oil red $\mathrm{O}$, destroys their physical states, these techniques cannot be used to document in vivo lipid structure. So we set out to preserve lipids in their native state and to correlate the histological findings with the physical, chemical studies described earlier.

The technique is as follows. Fresh tissue or tissue fixed lightly in formalin, a process which we showed did not affect the physical states of lipid, was cut into rings, frozen quickly in liquid nitrogen, placed on a microtome and serial thin sections were lifted onto slides. These serial sections were used for a variety of different stains. Several were taken for hot stage polarizing microscopy and then the section was actually stained by hematoxylin \& eosin, so that correlations could be made between the morphology and the physical chemistry.

Figure 15 A-D are of a lesion from a ballooned rabbit ileac artery fed cholesterol (Fig. 15). Figure $15 \mathrm{D}$ is the $\mathrm{H}$ \& $\mathrm{E}$ stain, after we had already observed the lesion by polarizing microscope. It shows the adventitia, the media, and a very thickened intima containing foam cells and other material, and a few foam cells even in the media. The birefringent material in cells are cholesterol ester droplets (Fig. 16).

I now want to show you how we have used this 
The Physico-Chemical Properties of Lipids during the Development and Regression of Atherosclerosis

$20^{\circ} \mathrm{C}$

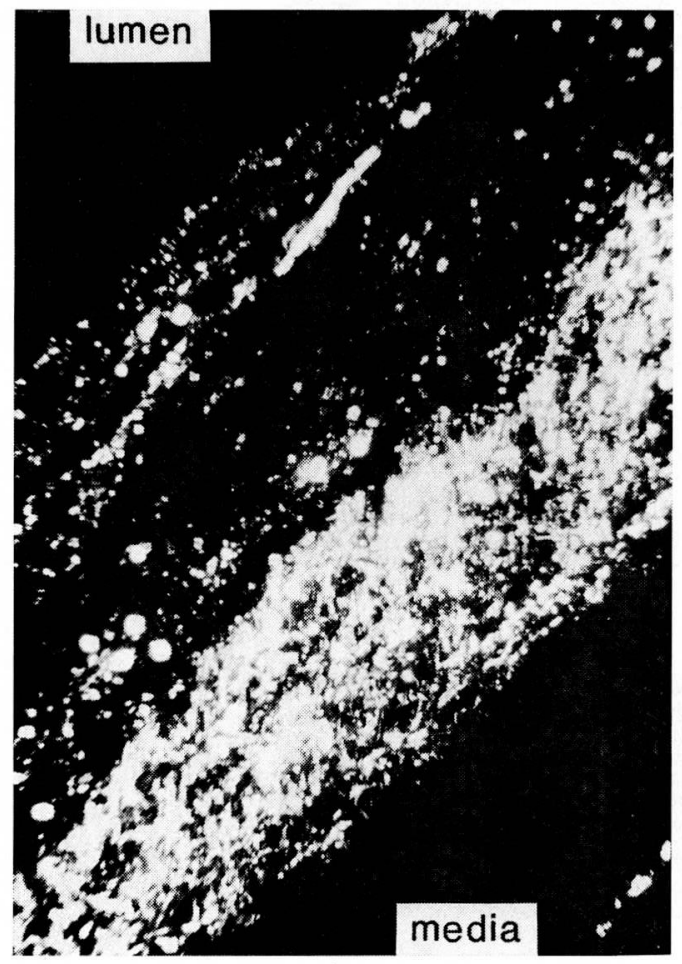

A

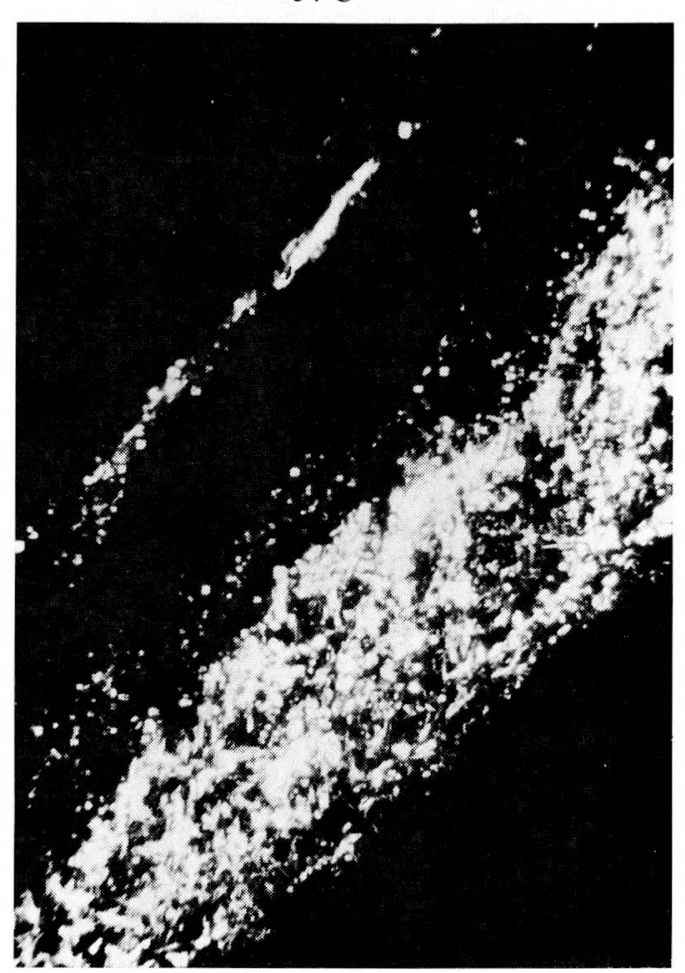

B

Fig. 18 Unfixed, thawed, frozen sections of an aortic plaque from a patient with familial homozygous hypercholesterolemia. The positions of the lumen and the media are indicated. A. Crossed polarized light at $20^{\circ} \mathrm{C}$. B. Crossed polarized light at $54^{\circ} \mathrm{C}$. The birefringence that has disappeared between $20^{\circ}$ and $54^{\circ} \mathrm{C}$ is due to cholesterol esters; the remaining birefringence is largely due to cholesterol monohydrate crystals. The remaining droplets just above the mass of crystals are due to the phospholipid-rich phase (Zone I, Fig. 7). The birefringent streak below the media in lower right-hand corner at $20^{\circ} \mathrm{C}$ is due to triglyceride crystals in the adventitia. $\times 112$.

method to study the lipid anatomy of plaques obtained from a child who died from familial homozygous hypercholesterolemia. The patient was studied by Dr. Ahrens and his group at the Rockefeller hospital and the clinical history is given in Fig. 17.

He was born in 1974 and died late in 1983 . He was a receptor negative homozygote by Brown and Goldstein's receptor binding techniques. He was noted to have xanthomata at the age of one year. His cholesterol was measured because he had xanthomata and was found to be in the range of 700 to $900 \mathrm{mg} / \mathrm{d} l$. He was started on diet, cholestyramine, clofibrate, and niacin. And over these next several years his serum cholesterol did not change appreciably. At age 4 he was noted to have arcus senilis, aortic stenosis, and he developed what turned out to be a myocardial infarction at age 4 . He was hospitalized and shortly after was given radioactive cholesterol to measure his cholesterol pool sizes. His body pool was 4.12 grams per $\mathrm{kg}$ of cholesterol with the total of 76 grams. Following this study he was given a portacaval anastomosis because at that time it was felt that it might help his hypercholesterolemia. Over the next few months cholesterol did fall to around 460 . Nevertheless, his cholesterol remained too high. He was brought back in the hospital and another cholesterol die-away curve was obtained. It showed that his total body cholesterol had in 
動脈硬化 Vol. 18 No.6 1990
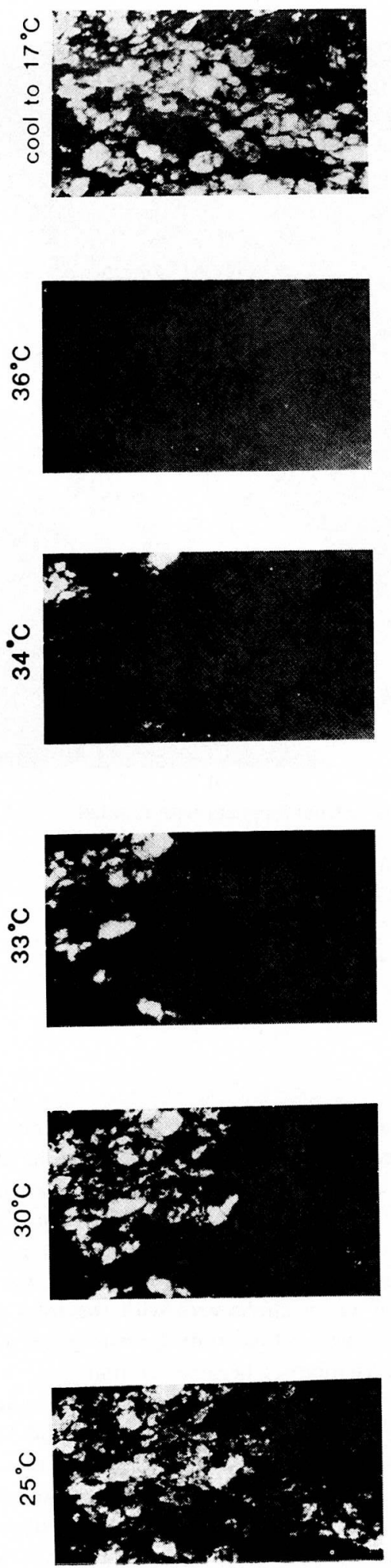

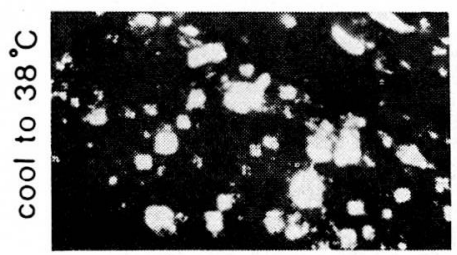

.

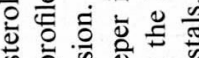

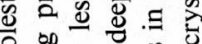
을 $\stackrel{0}{\Xi} \cong$

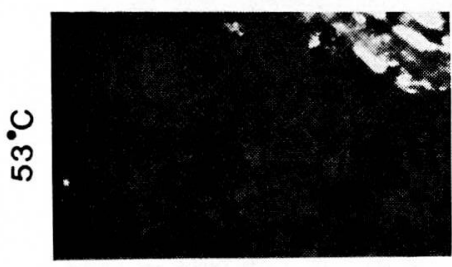
号 $\cong$ क्ष

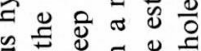

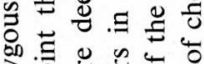
六 ¿

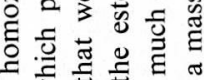

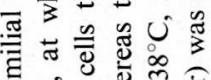

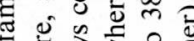

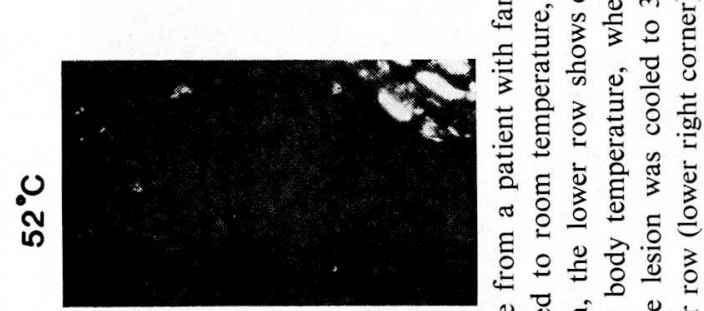

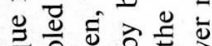
完

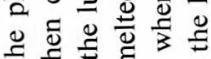

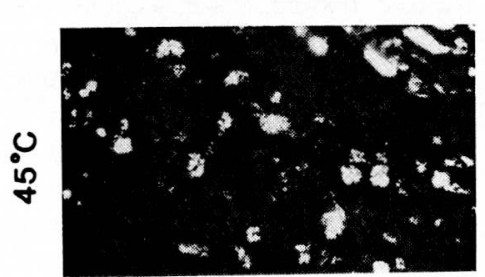
$\pm \cong \pm$ : 营 $0 \cong$ के प्ष

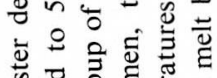
ช 芯 তั 岁引 引
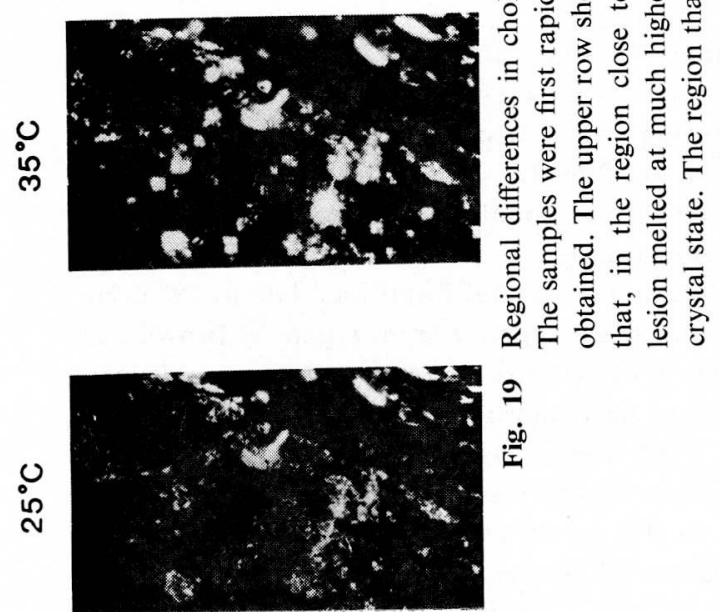
fact dropped to 42 grams but the serum cholesterol still remained unacceptably high. In the next few years he developed increasing angina and he was brought back into the hospital for study in late 1983. He was taken to cardiac catheterization because he developed accelerated angina. In that cardiac catheterization he developed a myocardial infarction and died.

Shortly after death we obtained aortic tissue from which we made frozen sections. The mean chemical compositions of four large plaques were: cholesterol $42 \pm 8$, cholesterol esters $35 \pm 8$, phospholipids $24 \pm 7$ (mean \pm SEM), a composition similar to plaques shown in Fig. 11. The histological appearance of his plaques were quite similar to those of adults.

A section of one plaque is shown under polarizing microscope, before staining in Fig. 18. Between $25^{\circ} \mathrm{C}$ and $54^{\circ} \mathrm{C}$ some of the material has melted. A massive amount of angular material at the base of the intima and a few droplets are left at $54^{\circ} \mathrm{C}$. Thus, what melted was the cholesterol ester and what remains is largely cholesterol crystals, the angular birefringence, and phospholipid in multi-lamellar phases.

Regional differences in cholesterol ester melting are shown in Fig. 19. In this plaque low melting cholesterol esters are present near the lumen and high melting esters (cholesteryl oleate and palmitate) are present adjacent to the mass of cholesterol crystals in the base of the plaque.

The crystals in this plaque are in positions in the hematoxylin and eosin stain section where clefts were present. Thus the clefts one sees on standard histological stains are due to cholesterol monohydrate crystals and not cholesterol ester crystals. In fact, needle-like clefts are occupied by cholesterol monohydrate plates. The reason they look needle-shaped is that if you cut through a thin plate it looks like a needle.

Thus, particular plaque and in others the foam cells towards the outer or the luminal side of the plaque are low melting, towards the bottom of the plaque the cholesterol esters are high melting, and at the base of the plaque we find many, many crystals.

\section{Progression of Lesions from Thickened Intima to Atheroma}

How do plaques form and grow? Since the patient-the same patient that we have talked

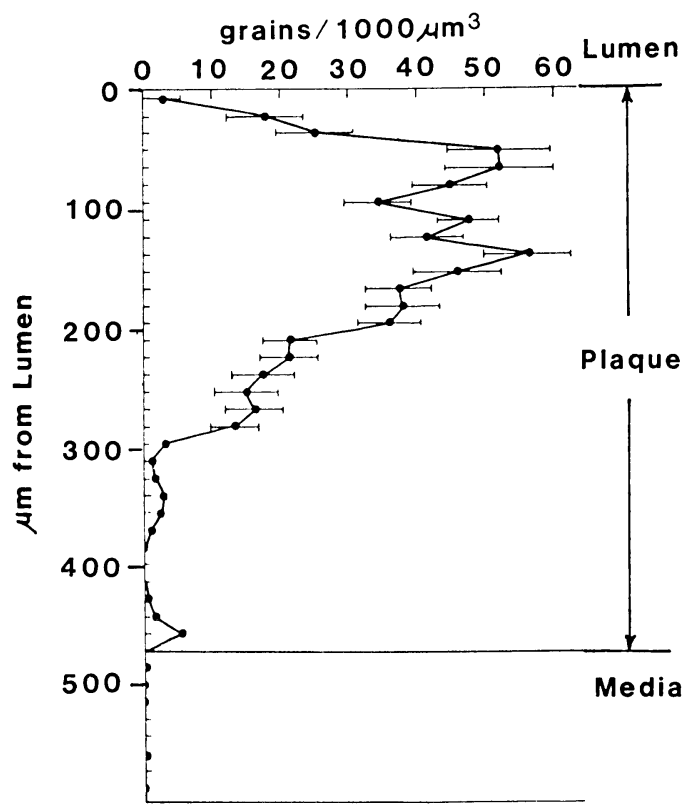

Fig. 20 Morphometrics of silver grain concentration in a plaque of the aortic wall of a patient with familial homozygous hypercholesterolemia (D. M. Small et al., unpublished). Groups of 10 to 15 squares $(6.6 \times 6.6 \mu \mathrm{m})$ were counted in sequential rows roughly parallel to the luminal border. Each row was plotted as a mean number of grains per square, expressed as mean grains per $1,000 \mu \mathrm{m}^{3} \pm \mathrm{SEM}$. The large SEM in the first $150 \mu \mathrm{m}$ of intima was due to grains being localized in foam cells. Almost no grains were seen in the base of the lesions despite many cholesterol crystals. Few grains were seen in the first $20 \mu \mathrm{m}$, the fibrous cap of the lesion.

about before (Fig. 17)-received tritium and carbon-14, in 1978 and again carbon-14 in 1981, we measured the radioactivity in the plaques. No tritium was found but ${ }^{14} \mathrm{C}$ counts were present. Curiously the specific activity of the free cholesterol in the plaque was only about a half of the cholesterol ester as if some unlabeled cholesterol had been deposited before the cholesterol esters. We used autoradiography on our frozen sections to see if we could locate the radioactive cholesterol. After incubating frozen sections with emulsion for about 3-6 months we were able to see silver grains in the frozen sections.

By taking this whole section and counting all of the grains per each $1,000 \mu \mathrm{m}^{3}$ from the luminal surface down through the media, we were able to 

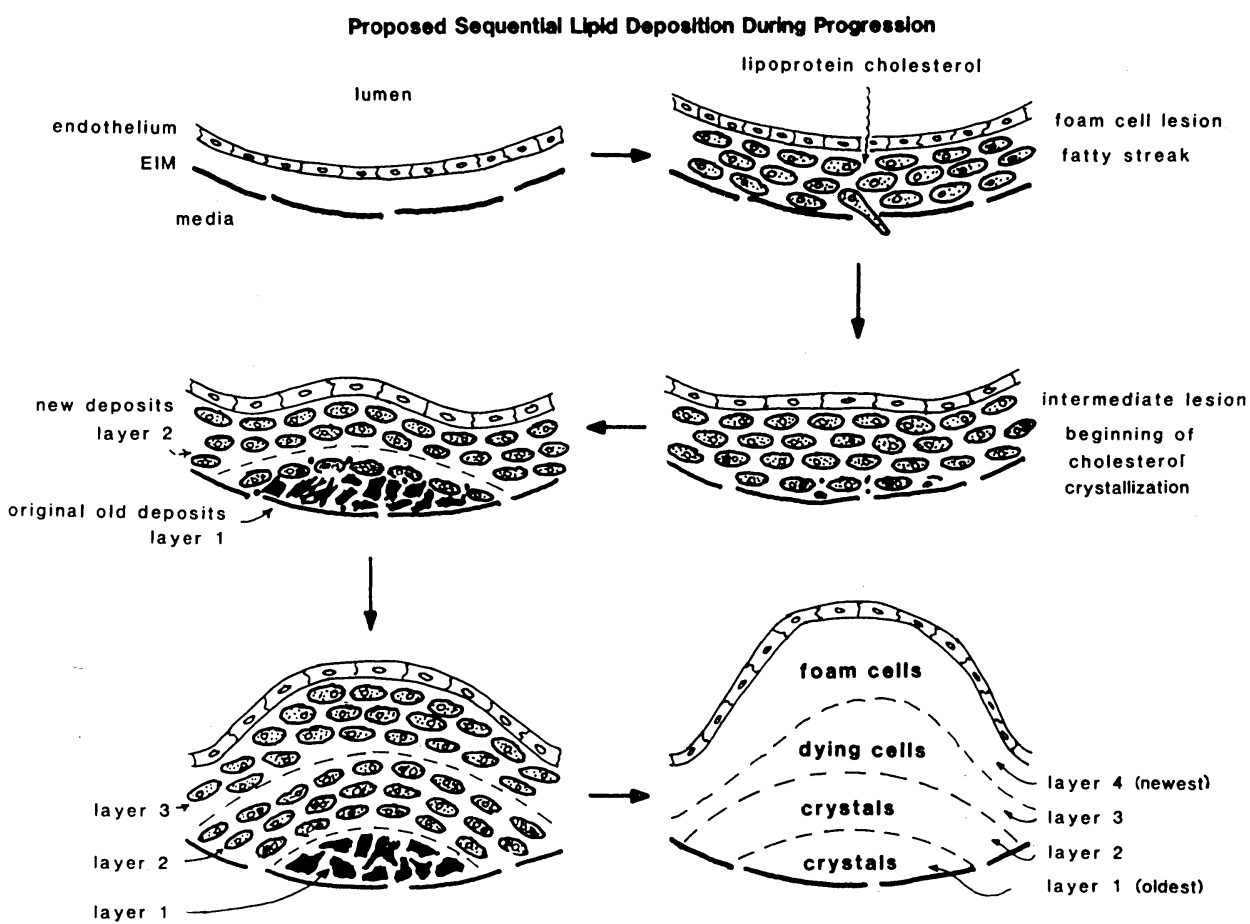

Fig. 21 Proposed stratification of sequential lipid deposits during progression. This sequence suggests that the normal intima (upper left) progresses through a series of stages (fatty streak, intermediate lesion) to an atheroma at the bottom. Cholesterol appears to come from the luminal side, with the newest deposits being just beneath the intima and the older deposits being deeper in the lesion.

construct a profile of the uptake of the cholesterol radioactivity into the vessel wall as a function of the depth. That profile is shown in Fig. 20.

The radioactive cholesterol in the plaque is concentrated in foam cells in the luminal lesions. Even though the deeper region is rich in cholesterol, it shows almost no autoradiographic silver grains. Thus cholesterol in the superficial and basal regions of the plaque does not exchange readily between parts of the plaque. These findings suggest that cholesterol is laid down sequentially in foam cells in the luminal side as cholesterol ester. As older deposits are buried more deeply in the layers, unsaturated cholesterol esters are hydro-

Fig. 22 Vector analysis of the compositional changes during regression. A. The chemical changes occurring during regression, mean $\pm \mathrm{SEM}$ intimal lipid composition. Both the 18-month (not shown) and the 24-month high cholesterol diet groups had lesions that had compositions like fatty streaks; however, at the 6-month regression time the lesion had a composition more like a plaque. B. The 6-month regression changes can be analyzed by two vectors, one describing the addition of cholesterol and the other, the subtraction of cholesterol ester. C. The 6-month to 12 -month changes can be described by vectors that indicate the subtraction of both cholesterol ester and free cholesterol. The absolute content of these lipids also decreases in the vessel wall, indicating true regression between 6 and 12 months. D. Chemical changes are related to the physical changes noted in the lesions. Since the total lipid content does not change greatly during the first 6 months of regression, it appears that cholesterol esters are converted into free cholesterol. Between 6 and 12 months, true regression is accompanied by a near absence of foam cells and cholesterol ester droplets and fewer crystals. 
The Physico-Chemical Properties of Lipids during the Development and Regression of Atherosclerosis

lyzed, making the composition richer in free cholesterol such that cholesterol crystals nucleate and deposit. Once cholesterol crystals deposit, they are quite inert and are resistant to removal even though the total body pool can be drastically reduced.
We propose the following process for the formation of atherosclerotic lesions (Fig. 21).

First, starting with the normal or slightly thickened intima, a high level of atherogenic lipoproteins enters the vessel wall. Cells are recruited from both blood and the media and these cells

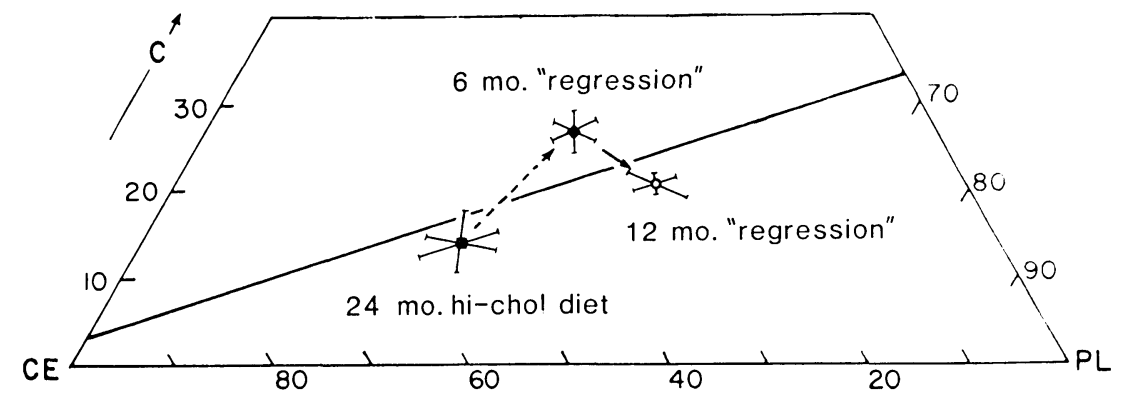

A

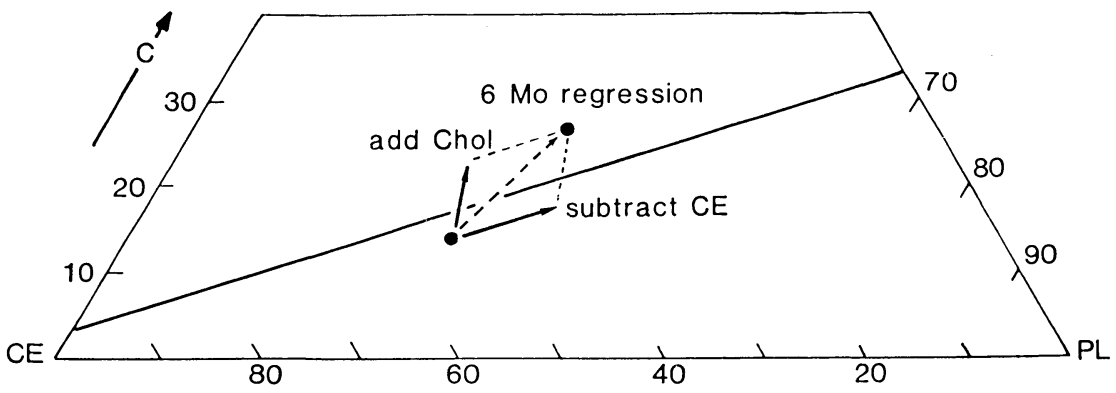

B

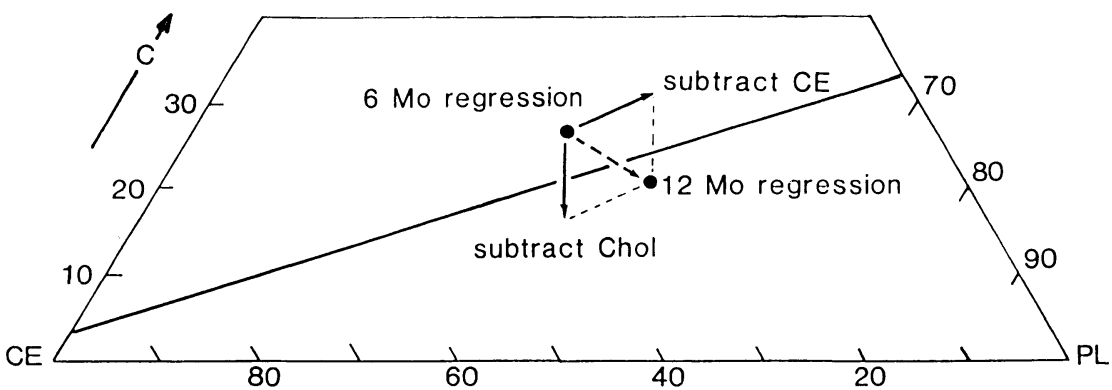

C

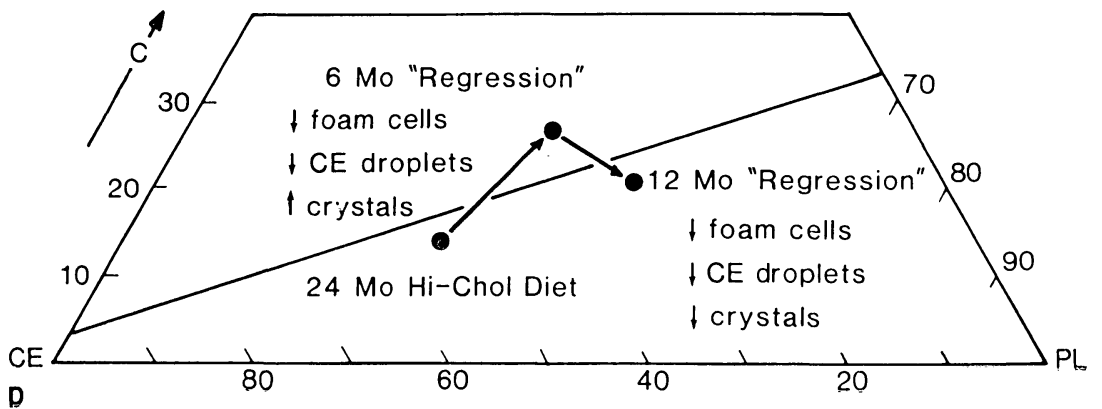


take up lipoproteins (perhaps oxidized, denatured and/or aggregated) and become foam cells, perhaps by one of the mechanisms that Dan Steinberg and colleagues have suggested. A cluster of these cells becomes a fatty streak. With time, some of the cells become supersaturated with cholesterol to initiate the formation of an intermediate lesion. Crystals may then nucleate and grow to cause cellular death and promote necrosis. Coincident with crystal deposition and necrosis at the base of the lesion, new cells are recruited to the upper part of the lesion just below the endothelium. This process continues to enlarge the lesion with new cells coming at the luminal surface and the older deposits further down. Further necrosis and crystal formation occur swelling the core. Thus the plaque atheroma consists of new cells at the luminal edge and the crystallized tombstones of older deposits at the base. Such simple atheroma then can become complicated with thrombofibrous cap formation, calcium apatite crystallization, ulceration and hemorrhage.

\section{Regression of Atherosclerotic Lesions}

Having proposed a sequential deposition from the luminal surface to form a chronologically stratified plaque, we would like to ask finally: Do plaques regress? And if so, how do they regress?

There is a large body of evidence in animal models to indicate that regression can occur if serum cholesterol is reduced to normal levels, that is below $160 \mathrm{mg} / \mathrm{d} l$. To study the physical-chemical changes occurring in lesions, we turned to the nonhuman primate model developed at Bowman Gray Medical School. Cynomolgus monkeys were fed a cholesterol containing diet for 18 months to induce fatty streak-intermediate like lesions. The diet was then changed to a low cholesterol diet and the cholesterol fell from $\sim 600 \mathrm{mg} / \mathrm{d} l$ to $150 \mathrm{mg} / \mathrm{d} l$ over a 3 month period. Animals were sacrificed at 6 months (6 mo. regression) and at 12 months (12 mo. regression).

The biochemical changes in lipid composition (Fig. 22A) at 6 and 12 months regression are analysed in Fig. 22.

As we move from the induction lesion to the 6 months regression lesion (Fig. 22B), we move along the dashed line. This dashed line may be described by two vectors, one along the arrow as a subtraction of cholesterol esters and another along the arrow as an addition of free cholesterol. Thus during the first six-month regression there is a relative decrease in cholesterol ester and a relative increase in free cholesterol. Since the total cholesterol in the lesion is not significantly reduced during this first six-month period, cholesterol ester must be converted to free cholesterol probably by hydrolysis of the esters. Lesion thickness does not significantly decrease in this period.

The vector analysis in going from 6 months to 12 months is different (Fig. 22C). It again can be described by two vectors, one in which cholesterol esters are subtracted and another in which free cholesterol is subtracted. During this interval the total lipid and total cholesterol content of the lesion decreases during this period indicating that

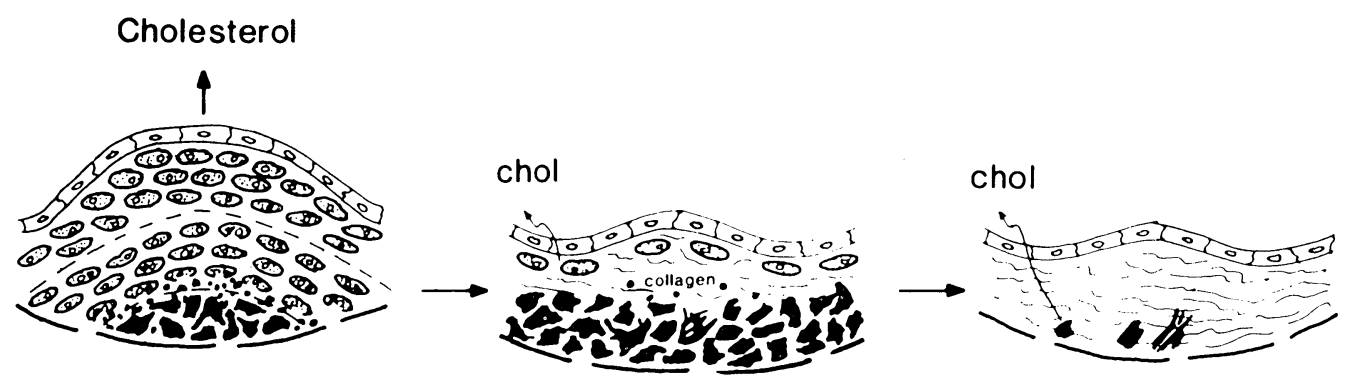

Fig. 23 Mechanisms of regression. During the early phase of regression, cholesterol influx appears to decrease, and foam cells and cholesterol ester droplets diminish. We suggest that the intermediate layers of foam cells undergo necrosis and that their cholesterol ester hydrolyzes and crystallizes to form more cholesterol crystals. This may lead to an increase in lesion thickness. With prolonged reduction of serum cholesterol, influx is greatly limited, and a gradual efflux of cholesterol from crystals occurs, leaving a plaque that has low lipid content but still contains collagen, calcium, and other plaque elements. 
these vectors adequately describe the movement of cholesterol out of the lesion. The lesion thickness significantly decreases during this period indicating true regression.

At 12 months regression there are still crystals present, even though they should not be present since the composition falls in a 2 phase region (See Fig. 7). This indicates that the dissolution rate of crystals into other phases or by HDL must be extraordinarily slow. This suggestion was bolstered by the extremely slow exchange of radioactivity of cholesterol between plasma and crystals as mentioned earlier.

Thus, in summary, the general sequence to the removal of lipids during regression is shown in Fig. 23. In a developed atherosclerotic plaque containing foam cells, extracellular esters and some crystals, a marked decrease in the serum cholesterol slows the influx of atherogenic lipoproteins into the lesion. This is accompanied by hydrolysis of some of the cholesterol ester droplets and the loss of most of the foam cells, an increase in free cholesterol and an increase in crystalline deposits.
It is probable that the cholesterol esters are hydrolyzed to form crystals during this process and that removal of cholesterol from the lesion is very slow. This process in humans probably lasts for months to years, with little or no change in the lesion size, and only a change in the composition. Once the cholesterol esters have been largely removed and foam cells are gone from the lesion, then there is a very slow process of dissolution of the large cholesterol crystals left in the basic lesion, which is probably brought about by high density lipoproteins. During this process lesions gradually decrease in volume leading to true regression.

謝辞: 本論文中の図 1-6, 8-12,14, 15, 18, 20, 21, 2226, 28, 29 は Dr. D.M. Small の Arteriosclerosis 8: 103-129，1988の論文中の図を引用したもので，この 引用は論文の著者 Dr. Small と発行機関の American Heart Association の許可のもとに行ったものである. ここに著者および AHA に対して感謝の意を表する. 第21回日本動脈硬化学会総会会頭

内 藤 周 幸 